\title{
Ultimate Strength of Metals
}

\author{
Michael Chandross $\odot^{*}, \neq$ and Nicolas Argibay ${ }^{\dagger, \ddagger}$ \\ Material, Physical, and Chemical Sciences Center, Sandia National Laboratories, Albuquerque, New Mexico 87123, USA
}

(Received 14 January 2020; accepted 10 February 2020; published 25 March 2020)

\begin{abstract}
We present a theoretical model that predicts the peak strength of polycrystalline metals based on the activation energy (or stress) required to cause deformation via amorphization. Building on extensive earlier work, this model is based purely on materials properties, requires no adjustable parameters, and is shown to accurately predict the strength of four exemplar metals (fcc, bcc, and hcp, and an alloy). This framework reveals new routes for design of more complex high-strength materials systems, such as compositionally complex alloys, multiphase systems, nonmetals, and composite structures.
\end{abstract}

DOI: 10.1103/PhysRevLett.124.125501

Despite nearly a century of effort, beginning with Frenkel's seminal work in the 1920s [1], a general theoretical framework that directly links bond strength and the strength of polycrystalline metals remains an elusive and invaluable target. The design of more reliable, cost-effective and damage-tolerant materials for use in applications such as energy efficient vehicles, aircrafts, and renewable energy systems (e.g., wind turbine gearboxes) continues to drive research and development efforts where the strength of metals is a primary and limiting factor. A means of estimating an upper bound for the strength of an alloy (i.e., under tension or other applied mechanical loads) is crucial for determining the size and geometry of structures and necessary for optimization of parameters like strengthto-weight ratios. The increasing reliance on integrated computational materials engineering makes accurate predictions of materials properties invaluable. While most metals used in engineering applications are alloys, the details and atomic origins of the strength of pure metals are instrumental to a formal understanding of alloys.

Frenkel initially calculated the shear strength of metals by considering a periodic potential and arrived at a liming strength of $\tau_{\max }=G b / 2 \pi a \cong G / 10$, where $\tau_{\max }$ is the maximum shear strength, $G$ is the shear modulus, $b$ is the magnitude of the Burgers vector, and $a$ is the lattice constant [1]. It was quickly realized that no metal has a strength approaching this value, and this realization was crucial for the discovery of dislocations and their role in plasticity [2-4]. Frenkel's approach was later refined by Mackenzie [5,6] and Kelly [7] to a value of approximately $G / 30$ that provided a more reasonable estimate of strength. However, these estimates are inaccurate (see below) as

Published by the American Physical Society under the terms of the Creative Commons Attribution 4.0 International license. Further distribution of this work must maintain attribution to the author(s) and the published article's title, journal citation, and DOI. metals tend to fail at stresses far below these ideal strengths, except in extreme cases such as nanowires [8-11]. Other more recent models predict the maximum shear strength of fcc metals to be $2 \gamma_{\mathrm{SFE}} / b$ based on stacking fault energies $\gamma_{\mathrm{SFE}}$ that determine the splitting distance between partial dislocations [12].

Generally, the stress required to nucleate and propagate dislocations becomes larger as the grain size decreases, and this is the basis for the Hall-Petch relationship $\sigma=\sigma_{0}+k d^{-1 / 2}$, where $\sigma$ is the uniaxial yield stress, and $\sigma_{0}$ and $k$ are constants [13]. The corresponding shear stress $\tau$ is the deviatoric component of $\sigma$ giving $\sigma=\sqrt{3} \tau$. As the grain size decreases below a critical grain size $d_{c}$, there is a crossover in mechanisms [14] to grain boundary sliding (GBS). In other words, when intragranular deformation (dislocation activity) becomes too energetically costly, intergranular mechanisms dominate, and the stress required to shear grain boundaries becomes the measure of the maximum strength of a material. There is currently no theory to predict this value from materials properties alone without adjustable parameters.

There is a large body of literature, including early explanations by Rosenhain and Ewen [15], calculations by Kê [16] and Mott [17], and more recent work [18,19] that consider GBS to be viscous flow between grains. Specifically, when deformation occurs on timescales too short for diffusive (i.e., creep) mechanisms to be active, and grain sizes are too small to accommodate dislocation activity, dynamic motion of material in the grain boundaries is required. We show that it is possible to estimate the maximum strength for all metals, including face-centeredcubic (fcc), body-centered-cubic (bcc), hexagonal-closepacked (hcp), and alloys by modeling GBS as viscous flow. Specifically, we calculate the energetic cost for the creation of a continuous, slip-accommodating amorphous interface from crystalline material (e.g., grain interiors, stacking faults, low-angle grain boundaries, dislocation cell walls, etc.). In other words, this implies deformation 
accommodation by converting an ordered phase to an amorphous phase that is effectively equivalent to a continuous network of high-angle grain boundaries. As in Frenkel's model of diffusion [1], or Mott's for GBS [17], we consider the strain rate to be an activated process with an energy barrier that is effectively lowered by the application of stress,

$$
\dot{\varepsilon}_{\mathrm{GBS}}=\dot{\varepsilon}_{\mathrm{tot}} \exp \left(-\frac{\Delta F-\tau V^{*}}{k T}\right),
$$

where $\dot{\varepsilon}_{\mathrm{GBS}}$ is the component of strain rate that is associated with GBS [20], $\dot{\varepsilon}_{\text {tot }}$ the total (applied) strain rate, $\Delta F$ is the activation energy, $\tau$ is the shear stress, $V^{*}$ is the activation volume, $T$ is the temperature, and $k$ is Boltzmann's constant. Because we are considering viscous flow at the boundaries, we take the activation energy to be related to the heat of fusion $L$, as this represents the energy difference between crystalline and amorphous phases [17]. Specifically, we use

$$
\Delta F=\left(L \frac{\rho_{L}}{M}\right)\left(1-\frac{T}{T_{m}}\right) f_{g} V^{*}
$$

where $\rho_{L}$ is the density of the liquid at the melting temperature (used as an approximation of the amorphous density), $M$ is the atomic mass, $T$ is the temperature, $T_{m}$ is the melting temperature, and $f_{g}$ is the volume fraction of crystalline material in the grains that incurs an amorphization energy penalty. Note that to make this an atomic process, we are effectively converting the heat of fusion to an atomic heat of fusion by dividing by the approximate atomic volume in an amorphous phase (i.e., $\rho_{L} / M$ ). The activation volume for single atomic motions is taken to be $b^{3}$ [21], where $b$ is the magnitude of the Burgers vector (equivalent to a metallic atomic diameter), and the volume fraction $f_{g}=[(d-\delta) / d]^{3}$ is the same as in previous models [22,23], with $d$ the grain diameter and $\delta$ the grain boundary width. This term accounts for the decreasing amount of crystalline material that must be amorphized as the grain boundary density increases with decreasing grain size. It is important to note that this relationship is generally valid for any space-filling polyhedra [23]. It is also useful to note that the calculated amorphization energy is effectively equivalent to the energy of a high angle grain boundary, a concept similar to that proposed by Gottstein and Shvindlerman [24]. In Fig. 1 we present a comparison of comprehensive DFT-determined average values of grain boundary energies for several metals [25] to predicted values from our expression above, multiplied by an approximate grain boundary width $(2 b)$, $\gamma_{\text {HAGB,calc }}=\left[L\left(\rho_{L} / M\right)\right]\left[1-\left(T / T_{m}\right)\right](2 b)$. Both the DFT and predicted values are shown at zero kelvin, and atomic diameters of fully coordinated crystal structures were used in place of the magnitude of the Burgers vector.

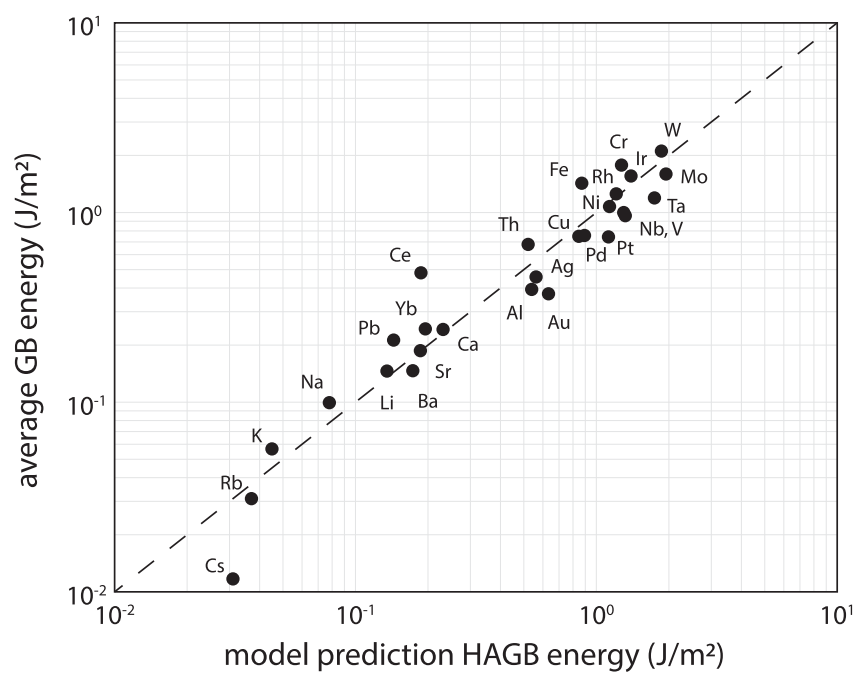

FIG. 1. Reference average values of grain boundary energy from DFT [25] compared to predicted values from heat of fusion at zero kelvin.

Expanding the exponential in Eq. (1) to first order and rearranging terms gives an expression for the stress,

$$
\tau=\left(L \frac{\rho_{L}}{M}\right)\left(1-\frac{T}{T_{m}}\right) f_{g}+\left(\frac{\dot{\varepsilon}_{\mathrm{GBS}}}{\dot{\varepsilon}_{\mathrm{tot}}}-1\right) \frac{k T}{b^{3}} .
$$

The ratio $\dot{\varepsilon}_{\mathrm{GBS}} / \dot{\varepsilon}_{\mathrm{tot}}$, which is difficult to determine experimentally, has been the subject of much discussion in the superplasticity literature [20]. However, noting the similarity of Eq. (3) to the empirical superplasticity equation for pure GBS $\dot{\varepsilon}=\left(\tau-\tau_{0}\right) / \eta_{\text {eff }}$, which treats grain boundaries as a Bingham fluid with a threshold stress $\tau_{0}$ and effective viscosity $\eta_{\text {eff }}$, we arrive at a form of Eq. (3),

$$
\begin{aligned}
\tau & =\tau_{0}+\eta_{\mathrm{eff}} \dot{\varepsilon}_{\mathrm{GBS}} \\
& =\left[\left(L \frac{\rho_{L}}{M}\right)\left(1-\frac{T}{T_{m}}\right) f_{g}-\frac{k T}{b^{3}}\right]+\left(\frac{k T}{\dot{\varepsilon}_{\mathrm{tot}} b^{3}}\right) \dot{\varepsilon}_{\mathrm{GBS}} .
\end{aligned}
$$

The final term accounts for viscous losses due to the shear of the boundaries. Considering that the viscosity of liquid metals is $\sim 1 \mathrm{mPa}$ s and experimental strain rates are sufficiently low that this term does not contribute strongly, we take it to be zero as a bounding case for our prediction. Equivalently, this implies that the ratio $\dot{\varepsilon}_{\mathrm{GBS}} / \dot{\varepsilon}_{\text {tot }}$ in Eq. (3) is small for experiments [20]. In dynamic simulations (i.e., not quasistatic, where there essentially is no applied strain rate), however, the ratio $\dot{\varepsilon}_{\mathrm{GBS}} / \dot{\varepsilon}_{\mathrm{tot}} \cong 1$, as all applied strain is directly transmitted to the boundaries. This causes the last term in Eq. (3) to become zero and gives a prediction for dynamic simulations of

$$
\tau=\left(L \frac{\rho_{L}}{M}\right)\left(1-\frac{T}{T_{m}}\right) f_{g} .
$$




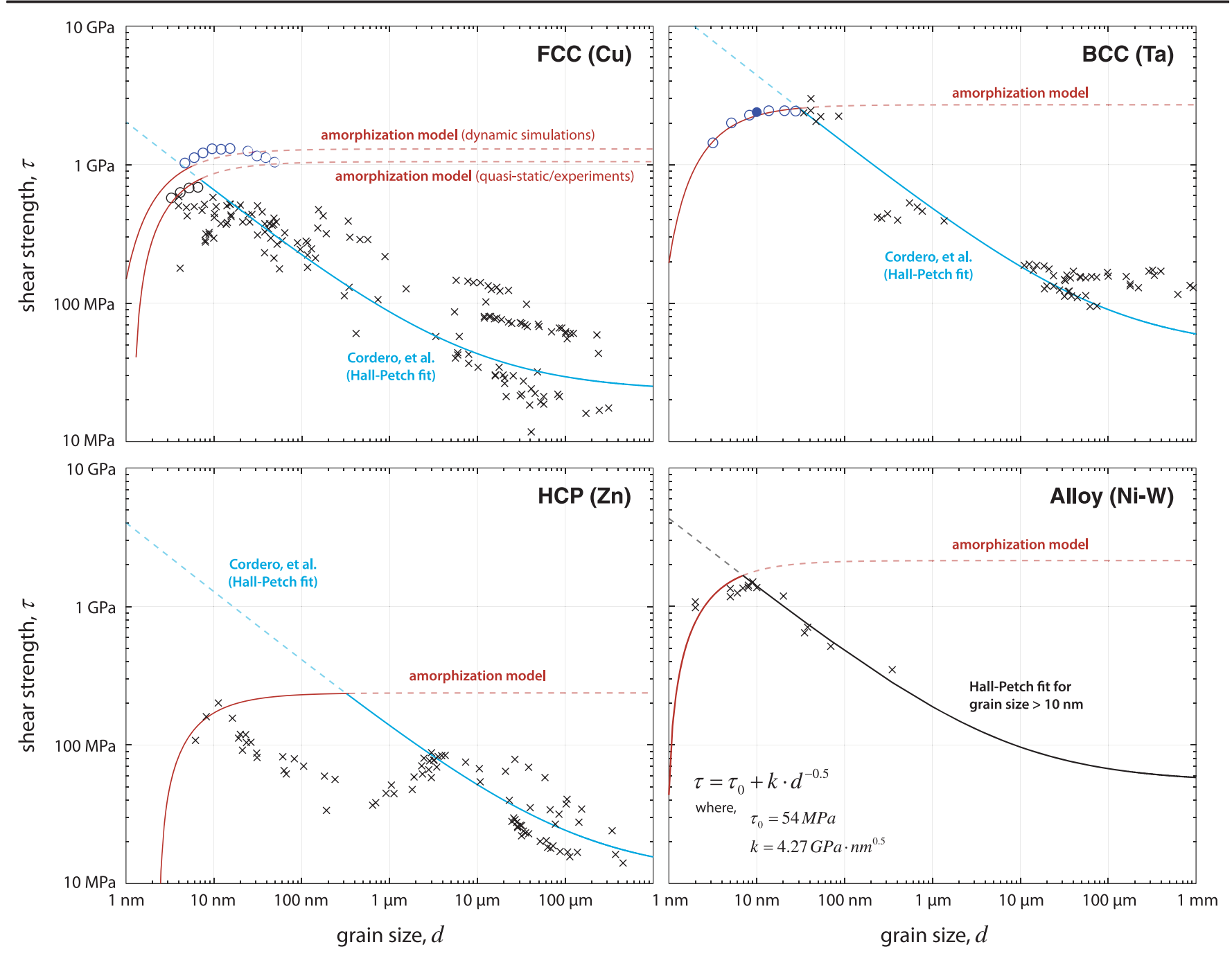

FIG. 2. Aggregate experimental $(x)$ and simulation $(o)$ data for exemplars of fcc $(\mathrm{Cu})[9,10,13,14]$, bcc $(\mathrm{Ta})[13,28,29]$, and hcp $(\mathrm{Zn})$ $[13,30]$ crystal structures, and an alloy (Ni-W) [26], the maximum shear strengths predicted using the amorphization model (red line), and corresponding Hall-Petch best fit (blue lines) from Cordero et al. [13] or from our fit to literature data (black line). Lines are solid or dashed to indicate regions where they are applicable (i.e., the mechanism with the lowest stress required to activate) or not applicable, respectively.

We show the (room-temperature) predictions of this model in Fig. 2, which require no adjustable parameters, along with published experimental and simulation data. All experimental data $(x)$ for pure metals in Fig. 2 are taken from the extensive Hall-Petch review from Cordero et al. [13], simulation data are shown as black and blue circles for quasistatic and dynamic cases, respectively, and for the alloy we also include a Hall-Petch equation best fit for grain sizes $>10 \mathrm{~nm}$ to the data from [26]. As data in the inverse Hall-Petch regime are scarce, we have chosen four representative cases (pure metals with fcc, bcc, and hcp crystal structures, and one alloy, Ni-W) to show the broad applicability of this model. A similar analysis for $\mathrm{Ni}$ is presented in the Supplemental Material [27]. In the cases where simulation data are available $(\mathrm{Cu}$ and $\mathrm{Ta}$; $\mathrm{Ni}$ in supplemental), we show predictions for both experimental and simulation conditions. Note that in all cases, the experimental predictions match experimental values very well, and in the case of $\mathrm{Cu}$, they also match the results from the quasistatic (i.e., $\dot{\varepsilon}_{\text {tot }} \rightarrow 0$ ) simulations of Schiøtz et al. [14]. With the correction for dynamic simulations, the predictions match high-strain rate results from Schiøtz and Jacobsen [9] for $\mathrm{Cu}$, and for Tang et al. [28] and Hinkle et al. [29] for Ta (filled circle).

With the remarkable agreement between the predictions and the available data, the possibility of predicting the ultimate strength of metals using this simple expression arises. The crossover between GBS and the Hall-Petch regime cannot currently be predicted for all metals, as there is only a theoretical prediction for fcc metals [12]. Instead, we note that the asymptotic value of our prediction (i.e., $f_{g}=1$ ) gives a reasonable upper bound for this strength. In Fig. 3, we show 


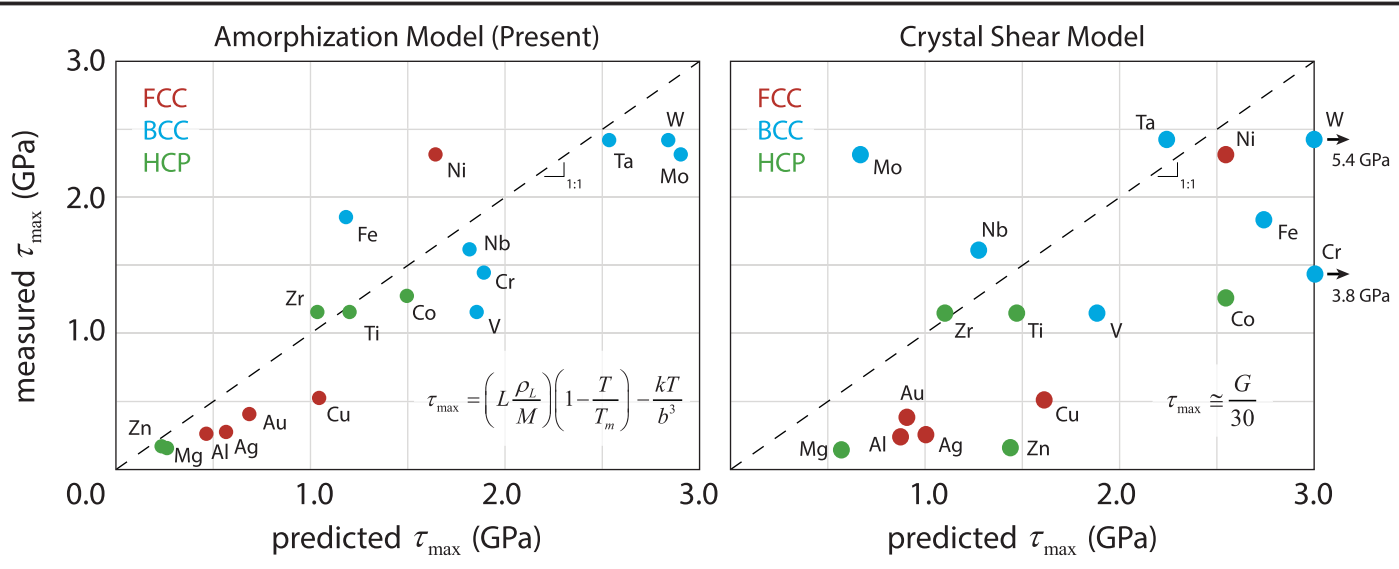

FIG. 3. Comparison of predicted ( $x$ axis) and measured ( $y$ axis) maximum shear strength for several metals. On the left are the predictions from the amorphization model presented here, while on the right are predictions from a model of crystal shear. The corresponding equations used to calculate predicted strengths are shown at the lower right of each plot.

the comparison of this prediction with the highest stresses shown in the Cordero et al. [13] review. Figure 3 shows that the amorphization model accurately predicts - without fitting - the maximum strength of a variety of metals, even with the caveats above that this prediction forms an upper bound. Almost all data in Fig. 3 show an obvious correlation with the prediction, and except in three cases, fall below the dashed line that indicates perfect agreement. We note that to arrive at a single value for experimental strength in Fig. 3, we used the highest reasonable stress from Cordero et al. [13] without consideration of additional strengthening mechanisms, such as from oxidation, as is possible in the cases of $\mathrm{Fe}$ and $\mathrm{Ni}$ that fall above the line. This forms an especially stringent test of the model. Also shown in Fig. 3 is the comparison of these strengths with the prediction of $G / 30$ from the Mackenzie model $[5,6]$. Agreement in this case is poor.

The success of this model in predicting the strength of metals leads to two major conclusions. The first is that in the absence or suppression of dislocation slip, the maximum strength of any metal is determined by the heat of fusion, and thus is a value that can be relatively easily calculated. The second implication is that it is possible to directly design alloys for maximum strength by calculating and optimizing the heat of fusion. This has strong implications in a variety of fields but seems particularly of value in the cases of high entropy or compositionally complex alloys [31], where the design space is highly multidimensional. The combination of this model with the regular solution model of Murdoch and co-workers [32] for stable, nanocrystalline binary alloys is also a tantalizing potential application for design of ultrastrong metals.

While considering GBS to be viscous flow with an energy barrier related to the heat of fusion is not a new concept, this model is the first to directly relate the strength of metals to materials properties without adjustable parameters or fitting. This implies a fundamental relationship between the strength of interatomic bonds (i.e., heat of fusion) and the strength of bulk, polycrystalline metals, linking nanoscale mechanisms to macroscopic properties. This theory thus serves to directly connect theoretical calculations with practical, experimental measurements and provides new insights to inform the design of higher-strength alloys.

The authors thank Thomas Hardin for providing insightful critical comments. This work was funded by the Laboratory Directed Research and Development program at Sandia National Laboratories, a multimission laboratory managed and operated by National Technology and Engineering Solutions of Sandia, LLC, a wholly owned subsidiary of Honeywell International, Inc., for the U.S. Department of Energy's National Nuclear Security Administration under Contract No. DE-NA0003525. Any subjective views or opinions that might be expressed in the paper do not necessarily represent the views of the U.S. Department of Energy or the United States Government.

* Corresponding author. mechand@sandia.gov Corresponding author. nargiba@sandia.gov

These authors contributed equally to this work.

[1] J. Frenkel, Z. Phys. 37, 572 (1926).

[2] E. Orowan, Z. Phys. 89, 605 (1934).

[3] F. C. Frank and W. T. Read, Phys. Rev. 79, 722 (1950).

[4] M. Polanyi, Z. Phys. 89, 660 (1934).

[5] N. H. MacMillan, J. Mater. Sci. 7, 239 (1972).

[6] J. K. Mackenzie, A Theory of Sintering and the Theoretical Yield Strength of Solids, Ph.D. Dissertation (University of Bristol, Bristol, 1949).

[7] A. Kelly, Strong Solids, 2nd ed. (Clarendon Press, Oxford, 1973).

[8] X. Li, Y. Wei, L. Lu, K. Lu, and H. Gao, Nature (London) 464, 877 (2010).

[9] J. Schiøtz and K. W. Jacobsen, Science 301, 1357 (2003). 
[10] L. Lu, X. Chen, X. Huang, and K. Lu, Science 323, 607 (2009).

[11] S. Wang, Z. Shan, and H. Huang, Adv. Sci. 4, 1 (2017).

[12] R. J. Asaro, P. Krysl, and B. Kad, Philos. Mag. Lett. 83, 733 (2003).

[13] Z. C. Cordero, B. E. Knight, and C. A. Schuh, Int. Mater. Rev. 61, 495 (2016).

[14] J. Schiøtz, F. D. Di Tolla, and K. W. Jacobsen, Nature (London) 391, 561 (1998).

[15] W. Rosenhain and D. Ewen, J. Inst. Met. 10, 119 (1913).

[16] T.-S. Kê, Phys. Rev. 71, 533 (1947).

[17] N. F. Mott, Proc. Phys. Soc. 60, 391 (1948).

[18] H. Conrad and J. Narayan, Scr. Mater. 42, 1025 (2000).

[19] H. Van Swygenhoven and A. Caro, Appl. Phys. Lett. 71, 1652 (1997).

[20] T. G. Langdon, J. Mater. Sci. 41, 597 (2006).

[21] H. Van Swygenhoven and A. Caro, Phys. Rev. B 58, 11246 (1998).

[22] H. W. Song, S. R. Guo, and Z. Q. Hu, Nanostruct. Mater. 11, 203 (1999).
[23] N. Wang, G. Palumbo, Z. Wang, U. Erb, and K. T. Aust, Scr. Metall. Mater. 28, 253 (1993).

[24] G. Gottstein and L.S. Shvindlerman, Grain Boundary Migration in Metals: Thermodynamics, Kinetics, Applications, 2nd ed. (CRC Press LLC, Baton Rouge, 2009).

[25] H. Zheng, X. G. Li, R. Tran, C. Chen, M. Horton, D. Winston, K. A. Persson, and S. P. Ong, Acta Mater. 186, 40 (2020).

[26] A. Giga, Y. Kimoto, Y. Takigawa, and K. Higashi, Scr. Mater. 55, 143 (2006).

[27] See Supplemental Material at http://link.aps.org/ supplemental/10.1103/PhysRevLett.124.125501 for model prediction and comparison to experimental data for nickel.

[28] Y. Tang, E. M. Bringa, and M. A. Meyers, Mater. Sci. Eng. A 580, 414 (2013).

[29] A. R. Hinkle, J. F. Curry, H. Lim, B. L. Nation, M. R. Jones, P. Lu, N. Argibay, and M. Chandross (in preparation).

[30] H. Conrad and J. Narayan, Appl. Phys. Lett. 81, 2241 (2002).

[31] D. B. Miracle and O. N. Senkov, Acta Mater. 122, 448 (2017).

[32] T. Chookajorn, H. A. Murdoch, and C. A. Schuh, Science 337, 951 (2012). 